\title{
Prediction of Visibility for Color Scheme on the Web Browser with Neural Networks
}

\author{
Miki Yamaguchi ${ }^{1}$ \\ School of Science for Open and Environmental Systems \\ Keio University, Kanagawa, Japan
}

\author{
Yoshihisa Shinozawa $^{2}$ \\ Faculty of Science and Technology \\ Keio University, Kanagawa, Japan
}

\begin{abstract}
In this study, we propose neural networks for predicting the visibility of color schemes. In recent years, most of us have accessed websites owing to the spread of the Internet. It is necessary to design web pages that allow users to access information easily. The color scheme is one of the most important elements of website design and therefore, we focus on the visibility of the background and character colors in this study. The prediction methods of visibility of color scheme have been proposed. In one of the prediction methods, neural networks are used to forecast pairwise comparison tables that indicate the visibility of background and character colors. Our model employs neural networks for color recognition and visibility prediction. The neural networks used for color recognition include functions that forecast the color class name from a color and extract the features of the color. The neural networks used for visibility prediction include functions that employ the features of background and character colors extracted by neural networks for color recognition and forecast the visibility of a color scheme. Pairwise comparison tables are forecasted with the prediction results of neural networks for visibility prediction. We conducted pairwise comparison experiment on a web browser, as well as color recognition experiment and evaluated our model. The results of the experiments suggest that our model could improve the accuracy of pairwise comparison tables compared to existing methods. Thus, proposed model can be used to predict the visibility of color schemes.
\end{abstract}

Keywords-Visibility prediction; color recognition; pairwise comparison experiment; human color vision; neural networks

\section{INTRODUCTION}

Websites on the Internet transmit and aggregate information. Therefore, they need to be created with consideration for content and ease of viewing (visibility). Elements that contribute to the visibility of a webpage include the color scheme, character size, and the overall layout, with the color scheme regarded as a particularly important aspect. This study focuses on the visibility of the background and character colors (henceforth referred to as the color scheme) displayed on a webpage.

As websites need to be designed considering color scheme visibility, standards or indices of ease of viewing are necessary. Consequently, the World Wide Web Consortium (W3C) recommends a brightness difference of at least 125 and a color difference of at least 500. However, an index that expresses the visibility of individual color schemes has not yet been devised. Therefore, many research attempts to clarify the color schemes of higher or lower visibility. Examples of these studies include attempts to clarify the color scheme visibility through experiments [1]-[5] and the development of software that can judge color scheme visibility or change the scheme to increase visibility. In particular, the proposed method involves the use of pairwise comparison to collect data on the visibility of background and character colors, and subsequently employing the Thurstone pairwise comparison method to index color scheme visibility [6]. As this type of research attempts to clarify the color scheme visibility through the collection and analysis of experimental data, a large volume of data are required. In this context, experimental data was used to develop predictive devices that can judge visibility levels and subsequently use them to predict the visibility of unfamiliar color schemes [7], [8].

The objective of this study is to use experimental data to develop a predictive device capable of judging color scheme visibility, and then devise a method for predicting the visibility of unfamiliar color schemes. Previous research [6] proposed the use of the Thurstone pairwise comparison method on data collected from pairwise comparison experiments to create pairwise comparison tables (pairwise comparison scores) as an index of color scheme visibility. Other studies [7], [8] attempted to predict pairwise comparison tables for background and character colors, which are used as an index of the visibility of unfamiliar color schemes.

A study [7] on background and character color visibility proposed a method for deriving pairwise comparison tables that involve training a neural network and then employing it to predict the visibility of a color scheme that was not learned by the network. In this process, multiple color coordinate systems (RGB, HSV, XYZ, L*a*b*) for each background and character color were used [7] as input features for the neural network. Furthermore, another study [8] introduced a model of human color perception (the Multi-Stage Color Model [9]) to reproduce the perceptual process, extract features that are factors related to visibility, and use these features to train a neural network in an attempt to improve the predictive precision with regard to color scheme visibility.

This study attempts to improve on previous research [7], [8] by training a neural network to make it capable of predicting color scheme visibility. This study does not utilize the features derived from color systems or existing color perception models while predicting color scheme visibility. We propose training a neural network capable of color recognition (color recognition refers to specifying the color name of a particular color), extracting features related to color scheme visibility, and then employing them in the prediction of color 
scheme visibility. The proposed method is used to predict the pairwise comparison tables for unfamiliar color schemes. The predictions are then compared with the predictions made using the methods based on previous research [7], [8] to evaluate the validity of the proposed method.

This paper is structured as follows. Section 2 presents a review of the existing literature in the field. Section 3 discusses areas for improvement in the existing literature. Section 4 outlines the proposed method for collecting experimental data and predicting the visibility of unfamiliar color schemes. Section 5 discusses the experiment to evaluate the validity of the proposed method by comparing it with those in the existing literature. Section 6 discusses the results of the evaluation experiment. Finally, Section 7 presents a summary of the study and outlines future research directions.

\section{LITERATURE REVIEW}

\section{A. Data Collection by Pairwise Comparison Experiment}

To collect data on the visibility of color schemes displayed on browsers, this study utilized the Thurstone pairwise comparison method, which indexed color scheme visibility [6]. The data collection method, which involved a pairwise comparison experiment developed from the existing literature, is discussed below.

In the first step, the following pairwise comparison experiment was performed on twenty participants (individuals aged in their twenties and thirties, with normal color perception).

Fig. 1 shows that the participants were asked to select the easier to view of two character strings of the same color grouping but with different luminance, written horizontally next to each other (right side/left side) on a browser with the same background color for both strings. Microsoft Internet Explorer was used as a browser and was displayed in the center of the monitor at a size that allowed both screens to be seen at roughly the same time. The character strings displayed were the same sentence that could be read from left to right. The same monitor was used throughout the entire experiment. All other experimental conditions (lighting, the positioning of the monitor with respect to the participant) were similar for all participants.

The colors used in the experiment were limited to web-safe colors. For the background, four colors were selected from each of the ten color groups (red, green, blue, cyan, magenta, yellow, and four other web safe colors). For the character colors, five colors were selected from each of the same groups. The RGB values for the background and character colors used are presented in Table I and Table II (web safe colors are abbreviated as WSC1, WSC2, WSC3, WSC4). Consequently, for the case where the background color grouping was red, the $\mathrm{R}$ parameter of the RGB value was varied over four grades, \#66, \#99, \#cc, and \#ff. When the character color grouping was red, the R parameter of the RGB was varied over five grades, \#33, \#66, \#99, \#cc, and \#ff. Fig. 2 shows the luminance of the background and character colors.

A total of 400 combinations were shown to each participant for pairwise comparison. When the character color was from the red grouping, for example, a combination of 40 background colors (10 color groupings $\times 4$ grades per grouping) and 10 character colors (two selections from the five grades within the red color grouping ${ }_{5} \mathrm{C}_{2}$ ). Given that there are ten character color groupings, the number of observations per pairwise comparison experiment per person was 4000. Pairwise comparisons were not made between characters from different color groupings, and background/character colors were displayed in random order for each participant. Accordingly, four thousand observations of the following data were collected for each participant.

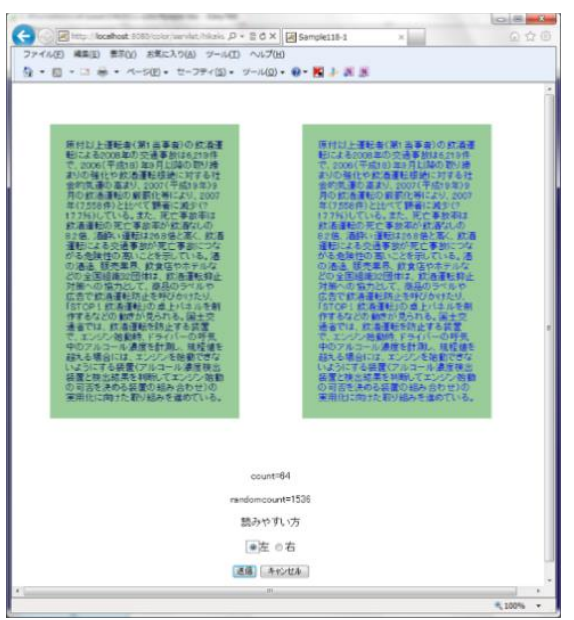

Fig. 1. Background Color and Character Color on the Browser.

TABLE. I. BACKGROUND COLORS

\begin{tabular}{|c|c|c|c|c|c|}
\hline \multicolumn{6}{|c|}{ Background Colors } \\
\hline$i$ & Color group & $j=1$ & $j=2$ & $j=3$ & $j=4$ \\
\hline 1 & Red & $\# 660000$ & \#990000 & $\# \mathrm{cc} 0000$ & \#ff0000 \\
\hline 2 & Green & $\# 006600$ & $\# 009900$ & $\# 00 \mathrm{cc} 00$ & $\# 00 \mathrm{ff} 00$ \\
\hline 3 & Blue & $\# 000066$ & $\# 000099$ & $\# 0000 \mathrm{cc}$ & \#0000ff \\
\hline 4 & Cyan & $\# 006666$ & $\# 009999$ & $\# 00 \mathrm{cccc}$ & $\# 00 \mathrm{ffff}$ \\
\hline 5 & Magenta & \#660066 & \#990099 & $\# \mathrm{ec} 00 \mathrm{cc}$ & \#ffooff \\
\hline 6 & Yellow & $\# 666600$ & \#999900 & $\# \operatorname{cccc} 00$ & $\#$ \#fffo0 \\
\hline 7 & WSC1 & \#ff6699 & \#ff9999 & \#ffcc99 & \#ffff99 \\
\hline 8 & WSC2 & $\# 669900$ & \#999900 & $\#$ \#cc9900 & \#ff9900 \\
\hline 9 & WSC3 & $\# 99 \operatorname{cc} 66$ & \#99cc99 & $\# 99 \mathrm{cccc}$ & \#99ccff \\
\hline 10 & WSC4 & \#666666 & \#996699 & $\#$ \#cc66cc & \#ff66ff \\
\hline
\end{tabular}

TABLE. II. CHARACTER COLORS

\begin{tabular}{|c|c|c|c|c|c|c|}
\hline \multicolumn{7}{|c|}{ Character Colors } \\
\hline$l$ & $\begin{array}{l}\text { Color } \\
\text { group }\end{array}$ & $l=1$ & $l=2$ & $l=3$ & $l=4$ & $l=5$ \\
\hline 1 & Red & $\# 330000$ & $\# 660000$ & \#990000 & $\# \mathrm{cc} 0000$ & \#ff0000 \\
\hline 2 & Green & $\# 003300$ & $\# 006600$ & $\# 009900$ & $\# 00 \mathrm{cc} 00$ & $\# 00$ ff00 \\
\hline 3 & Blue & $\# 000033$ & $\# 000066$ & $\# 000099$ & $\# 0000 \mathrm{cc}$ & $\# 0000 \mathrm{ff}$ \\
\hline 4 & Cyan & $\# 003333$ & $\# 006666$ & \#009999 & $\# 00 \mathrm{cccc}$ & \#00ffff \\
\hline 5 & Magenta & $\# 330033$ & $\# 660066$ & \#990099 & $\# \mathrm{cc} 00 \mathrm{cc}$ & \#ffooff \\
\hline 6 & Yellow & $\# 333300$ & $\# 666600$ & \#999900 & $\# \operatorname{cccc} 00$ & \#ffffo0 \\
\hline 7 & WSC1 & \#ff3399 & \#ff6699 & \#ff9999 & \#ffcc99 & \#ffff99 \\
\hline 8 & WSC2 & $\# 339900$ & $\# 669900$ & \#999900 & \#cc 9900 & \#ff9900 \\
\hline 9 & WSC3 & $\# 99 \mathrm{cc} 33$ & $\# 99 \operatorname{cc} 66$ & \#99cc99 & $\# 99 \mathrm{cccc}$ & $\# 99 \mathrm{ccff}$ \\
\hline 10 & WSC4 & $\# 336633$ & \#666666 & \#996699 & \#cc66cc & \#ff66ff \\
\hline
\end{tabular}




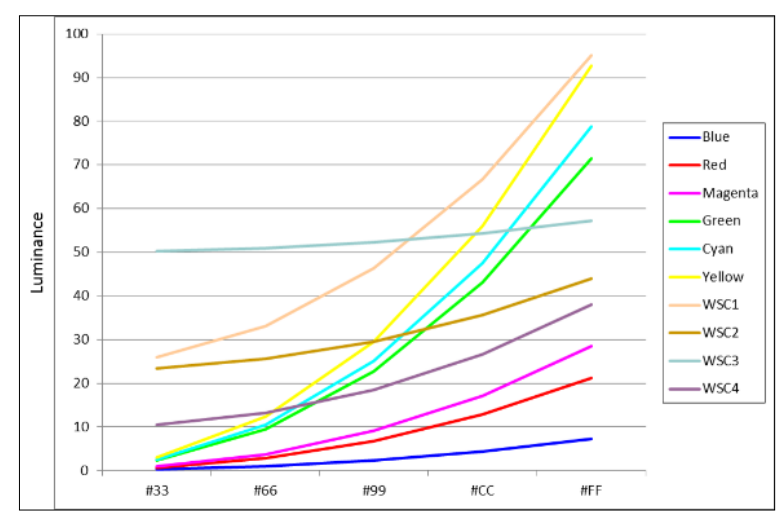

Fig. 2. Luminance of Background and Character Colors.

- Background Color, Left Character Color, Right Character Color, Direction that was easier to view

- \#330000, \#000066, \#000099, Left

\section{B. Thurstone Pairwise Comparison Method}

In the next step, the Thurstone pairwise comparison method was used to score the visibility of the background and character colors. In the pairwise comparison experiment, the luminance of the background colors in the $i$ grouping $(i=1,2, \ldots, 10$, where $i=1$ is red, $i=2$ is green, ..., $i=10$ is WSC4) was varied over four grades. The change in the luminance value was represented by $j(j=1,2,3,4)$ (see Table I). For example, for background colors in the red grouping $(i=1), j=1$ is \#660000, $j=2$ is \#990000, $j=3$ is \#cc0000, and $j=4$ is \#ff0000 (expressed as background color $j$ in color grouping $i$ ). Similarly, the luminance of the character color in the $k$ grouping $(k=1,2, \ldots$, 10 , where $k=1$ is red, $k=2$ is green, ..., $k=10$ is WSC4) was also varied over five grades (see Table II). The change in the luminance of the left character color is represented by $l(l=1,2$, $\cdots, 5)$ and the change in the luminance of the right character color is represented by $r(r=1,2, \cdots, 5, l \neq \mathrm{r})$ (expressed as character color $l$ or $r$ in color grouping $k$ ).

Accordingly, when participant $p(p=1,2, \ldots, 20)$ is presented with background color from group $i$ with the $j^{\text {th }}$ luminance value (background color $j$ from color grouping $i$ ) with the character color from group $k$ with the $l^{\text {th }}$ luminance value as the left-hand character color (character color $l$ from color grouping $k$ ) and the character color from group $k$ with the $r^{\text {th }}$ luminance value as the right-hand character color (character color $r$ from color grouping $k$ ), the value of the visibility of the color scheme will be $x_{p i j k l}$. When participant $p$ judges the right character color as easier to view, $x_{p i j k l}=1$ and when the left character color is judged as easier to view, $x_{p i j k l}=0$. The mean value of all twenty participants will be $y_{i j k l r}$ (Equation (1)).

$y_{i j k l r}=\frac{1}{20} \sum_{p=1}^{20} x_{p i j k l r}$

Specifically, for the background color $j$ in the color group $i$, $y_{i j k l r}$ will be the ratio of cases where the right-hand character $r$ in color grouping $k$ is judged to be easier to view than the lefthand character $l$ from the same color grouping. Accordingly, $y_{i j k l r}=1.0-y_{i j k r l}$ (in the case where $i=r, y_{i j k l}=0.5$ ). Next, the inverse function of the cumulative distribution function of the standard normal distribution is derived with respect to $y_{i j k l r}$
(Normsinv in Equation (2)). Subsequently, the mean values for all character colors are calculated, and the pairwise comparison scores $z_{i j k r}$ are derived (Equation (2)).

$z_{i j k r}=\frac{1}{5} \sum_{l=1}^{5} \operatorname{Norm} \operatorname{sinv}\left(y_{i j k l r}\right)$

For the background color $j$ in color group $i$, the pairwise comparison score $z_{i j k r}$ will be the index of the visibility of character color $r$ in color group $k$.

According to the above process, the experimental data, for the ten background color groupings for each character color, 40 observations per person (4 grades of background color $\times 10$ character color pairs) was employed to quantify the visibility levels of the combinations of the four background colors and five character colors. As an example, Table III presents the results for the case where the character color was from the red grouping, and the background color was from the green grouping.

The columns in Table III display the RGB values of the background colors (4 colors) and the rows display the RGB values of the character colors (5 colors). Higher pairwise comparison scores indicate color schemes that are easier to observe and vice versa, which yielded the number of background colors for each character color, ten pairwise comparison tables, for a total of 100 tables.

TABLE. III. RESUlTS OF THURSTONE PAIRWISE COMPARISON METHOD (CHARACTER COLOR RED, BACKGROUND COLOR GREEN)

\begin{tabular}{|l|l|l|l|l|l|}
\hline \multirow{2}{*}{$\begin{array}{l}\text { Background } \\
\text { colors }\end{array}$} & \multicolumn{5}{|l|}{ Character colors } \\
\cline { 2 - 6 } & $\# 330000$ & $\# 660000$ & $\# 990000$ & $\# c c 0000$ & $\# f f 0000$ \\
\hline$\# 006600$ & 0.157 & -0.497 & -0.714 & 0.221 & $\# 0.833$ \\
\hline$\# 009900$ & 1.006 & 0.408 & -0.418 & -0.769 & -0.228 \\
\hline$\# 00 \mathrm{cc} 00$ & 1.064 & 0.633 & -0.156 & -0.730 & -0.810 \\
\hline$\# 00 f f 00$ & 1.077 & 0.573 & 0.0 & -0.573 & -1.077 \\
\hline
\end{tabular}

\section{Predicting the Visibility of a Color Scheme}

If it were possible to predict the pairwise comparison tables even for unfamiliar color schemes, then these tables could be used to determine whether or not the combination of background and character colors on the webpage being currently viewed by the user is easy or difficult to view. Furthermore, if the color scheme was judged to be difficult to view, it would be possible to change it to a scheme that is easier to view by switching it to a combination of higher value. If this kind of feature could be implemented in a browser, it would be possible to determine the visibility of the page currently being viewed and to change it to a color scheme with higher visibility.

In this context, a previous study [7] proposed a method whereby a portion of the data collected from a pairwise comparison experiment is employed as color scheme data, with the remainder treated as unfamiliar data with a neural network (henceforth "network") used to predict its pairwise comparison tables. For example, only the data where the character color is from the red, blue, and green groupings are used, with the pairwise comparison tables for character colors from the remaining seven color groupings being predicted (here, the character color groupings employed in the training of the 
network are referred to as the basic color groupings). Consequently, the method attempts to teach the network using the basic color grouping data and then derive the pairwise comparison tables after using the trained network to predict the visibility of the unfamiliar color schemes.

Additionally, a previous study [7] used RGB, HSV, XYZ (Yxy) and $\mathrm{L}^{*} \mathrm{a}^{*} \mathrm{~b}^{*}$ color system values for each of the background and character colors as input features for the network to predict the color scheme visibility. Furthermore, luminance difference and the color difference between the background and character colors may influence the visibility of the color scheme. In this context, a previous study [8] introduced a model of human color perception (Multi-Stage Color Model [9]) to reproduce the color perceptual process, extract features that are factors related to visibility, and use these features to train a neural network in an attempt to improve the predictive precision of color scheme visibility.

\section{Overview of the Multi-Stage Color Model}

This section presents an overview of the Multi-Stage Color Model (henceforth, MSC Model) [9]. The MSC Model is a color perception model comprised of a number of stages.

In the first stage of the MSC Model, visible light (RGB values) are converted into response values (spectral sensitivity values) for three types of cones (L, M, and S).In this study, RGB values (gamma-corrected, as the pairwise comparison experiment, carried out on a computer display) were transformed into XYZ values and then, Equation (3) was used to derive the response values $L, M, S$ for each cone [10].

$$
\left(\begin{array}{c}
L \\
M \\
S
\end{array}\right)=\left(\begin{array}{ccc}
0.155 & 0.543 & -0.0329 \\
-0.155 & 0.457 & 0.0329 \\
0.0 & 0.0 & 0.016
\end{array}\right)\left(\begin{array}{l}
X \\
Y \\
Z
\end{array}\right)
$$

In the second stage, the response values of the opponent color cells are derived. There are opponent color cells that are stimulated by one cone and inhibited by the surrounding cones (on-center/off-surround type) and cells that are inhibited by one cone and stimulated by the surrounding cones (off-center/onsurround type). A total of six types of opponent color cells ON-center/OFF-surround type cells centered on the L-cone (with a response value denoted by $L_{o}$ ), the M-cone $\left(M_{o}\right)$, and the S-cone $\left(S_{o}\right)$ and OFF-center/ON-surround type cells centered on the L-cone $\left(-L_{o}\right)$, the M-cone $\left(-M_{o}\right)$, and the Scone $\left(-S_{o}\right)$. The three types of cones are randomly connected to these cells in a ratio assumed to be 10:5:1. Furthermore, the ratio of the response values of the center and surrounding cells is assumed to be 1:1. The response values for all opponent color cells are expressed in Equation (4).

$$
\begin{aligned}
& L_{0}=16 L-(10 L+5 M+S)=6 L-5 M-S \\
& M_{0}=16 M-(10 L+5 M+S)=-10 L+11 M-S \\
& S_{0}=16 S-(10 L+5 M+S)=-10 L-5 M+15 S \\
& -L_{0}=-16 L+(10 L+5 M+S)=-6 L+5 M+S \\
& -M_{0}=-16 M+(10 L+5 M+S)=10 L-11 M+S \\
& -S 0=-16 S+10 L+5 M+S=10 L+5 M-15 S
\end{aligned}
$$

In the third stage, the responses of the six types of color opponent cells are combined to set the way the color is seen. The basic combinations are the responses of $L_{o}-M_{o}$ and $M_{o}-$ $L_{o}$ and adding or subtraction the $S_{o}$ response will yield the response value that corresponds to the perception of red, yellow, green, and blue.

- $\operatorname{Red}\left(R_{o}\right)$ is formed by $L_{o}-M_{o}$ plus $S_{o}$.

- Yellow $\left(Y_{o}\right)$ is formed by $L_{o}-M_{o}$ minus $S_{o}$.

- Green $\left(G_{o}\right)$ is formed by $M_{o}-L_{o}$ minus $S_{o}$.

- Blue $\left(B_{o}\right)$ is formed by $M_{o}-L_{o}$ plus $S_{o}$.

The number of each type of cell is considered in the addition and subtraction process. In the MSC Model, the ratio of $L_{o}: M_{o}: S_{o}$ is assumed to be 10:5:2 with the color response being derived by Equation (5).

$$
\begin{aligned}
& R_{0}=10 L_{0}-5 M_{0}+2 S_{0} \\
& Y_{0}=10 L_{0}-5 M_{0}-2 S_{0} \\
& G_{0}=5 M_{0}-10 L_{0}-2 S_{0} \\
& B_{0}=5 M_{0}-10 L_{0}+2 S_{0}
\end{aligned}
$$

Thus, the luminance value $V$ is derived from Equation (6) and concludes the process in the MSC Model.

$V=L+M+S$

\section{PROPOSAL}

The aim of this study is to improve the existing research [7], [8] and increase the accuracy of visibility prediction by developing, through training, a network capable of predicting color scheme visibility. A previous study [8] used an existing color perception model while extracting features for visibility from background and character colors. Instead of using an existing model, in this study, data are collected from the color recognition experiment detailed in Section 4.2 and attempts to develop, through training, a color recognition network and then utilize it to extract features from background and character colors. Furthermore, this study proposes to use the extracted features to predict the color scheme visibility. Finally, the validity of the proposed method is evaluated by predicting the pairwise comparison tables for unfamiliar color schemes and then comparing them and their predictive precision with the tables predicted by existing methods [7], [8].

\section{A Method for Predicting the Visibility of Color SCHEMES}

\section{A. Overview of the Proposed Method}

Fig. 3 presents an overview of the proposed method, which consists of the color recognition neural network for feature extraction (henceforth color recognition network), the visibility prediction neural network for predicting color scheme visibility (henceforth visibility prediction network), and the pairwise comparison table prediction element. This section gives an overview of the proposed method, and further subsections provide details on its respective parts. The first aspect to be discussed is the color recognition network (see Section 4.3 for details). 


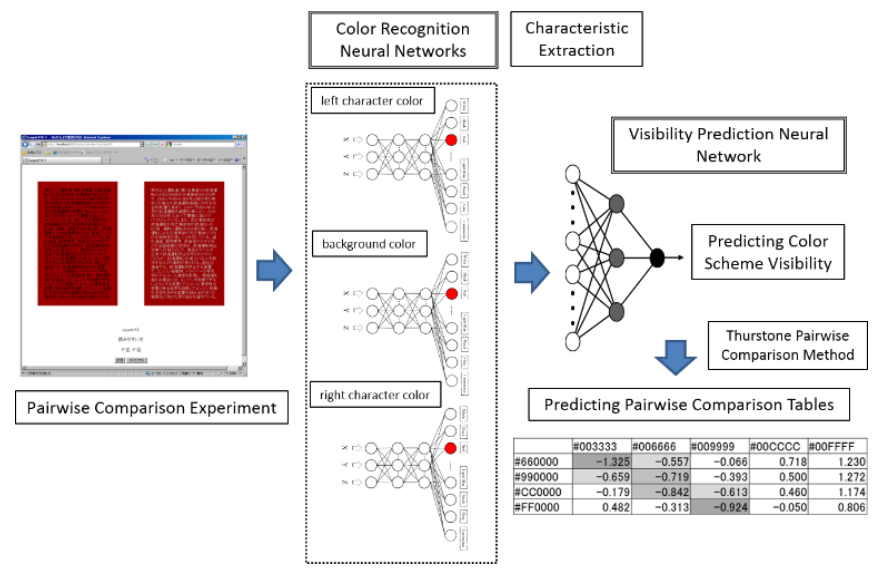

Fig. 3. Overview of the Proposed Method.

The existing color perception model employed in previous studies (MSC Model) has the ability to output the response value, which corresponds to the degree of luminance of the opponent color (red-green, blue-yellow) or the colors red, green, blue, or yellow, from the response value of the cone relating to an arbitrary color. To give the network in this study the same functionality as the existing color perception models, it was given the ability to output the color name and brightness of a particular color when its XYZ color system value is inputted. Thirteen colors that could be predicted were specified, white, black, red, green, yellow, orange, blue, purple, brown, yellow-green, light-blue, peach, and gray. Data concerning colors and their respective color names were collected through the color recognition experiment detailed in Section 4.2.

When predicting the visibility of a color scheme, three color recognition networks are prepared, the XYZ values for the background color and left/right character colors are entered, and the network is activated. The features to be entered into the visibility prediction network in the next step are then extracted. After the features of background color and left/right character colors extracted from the color recognition network are entered into the visibility prediction network, a prediction on which of the character colors has a higher level of visibility is made (see Section 4.4 for details). This network utilizes the three layer type neural network employed in the existing literature [7]. By combining these two network forms, the visibility of an unfamiliar color scheme can be predicted. In the element that predicts the pairwise comparison tables, the Thurstone pairwise comparison method is employed based on the results of the visibility prediction network to predict the pairwise comparison tables (see Section 4.5).

\section{B. Color Recognition Experiment}

To develop a color recognition network, a color recognition experiment that collected data on colors and respective color names was carried out. The color recognition experiment involved showing color sample cards to participants to match colors with color names. The color sample cards used in this study were from the DIC Color Guide $19^{\text {th }}$ Edition (652 colors) [11]. Sixty participants (aged in their twenties and thirties, with normal color perception) were involved. Participants were shown one card from the color guide and asked to match it with one of thirteen color names (white, black, red, green, yellow, orange, blue, purple, brown, yellow-green, light-blue, peach, and gray). Berlin \& Kay [12] defined eleven basic colors (white, black, red, green, yellow, orange, blue, purple, brown, peach, and gray). Furthermore, yellow-green and lightblue are often included in the colors that are generally perceived by Japanese people. Considering that the lack of colors can lead to difficulties in color recognition, too many will lead to the emergence of individual differences. This study included yellow-green and light-blue to make a total of thirteen colors. The XYZ values of the color sample cards were measured using a spectrophotometer (Konica Minolta CM700d). At the time of data collection, the color sample cards were shown to the participants under fixed lighting (approximately 400 lux) in front of a stable background (white with an $X Y Z$ value of $X=68, Y=72, Z=71$ ).

\section{Training the Color Recognition Neural Network}

Using data collected from the color recognition experiment, a network capable of predicting the color name of an unfamiliar color was developed. The structure of this network is shown in Fig. 4. The color recognition network was set up as a four layer feedback type because the MSC Model involves inputting an XYZ value (LMS cone response value) and outputting the color name after passing through the opponent color response. The input value was the XYZ color system value of the color sample card (the number of neurons in the input layer was three). The output value was the color name and luminance value (Y-value) of the color that was inputted (there were 14 neurons in the output layer). Based on the preliminary experiment, the number of neurons for the first and second middle layers was set at twenty and all layers were connected.

For a given color sample card, the XYZ value of the color was the input, and a teaching signal was applied to the color name neuron to correspond to the ratio of the responses of the participants in the color recognition experiment. When the number of participants that responded "Blue" was 45 and "Light Blue" 15 for example, 45/60 was applied to the neuron corresponding to blue in the output layer, 15/60 was applied to the neuron corresponding to light blue and 0 was applied to the other neurons. For the luminance value, the Y-value was applied as the teaching signal. In this way, the 652 color sample cards were used as training data to train the color recognition network to output the teaching signals. The training algorithm is as follows.

1) Randomize the number of connections inside the color recognition network.

2) Training data $\mathbf{x}_{\mathbf{i}}(\mathrm{XYZ}$ color system) (i=1,2, $\cdots, 652$, $\mathrm{XYZ}$ value is normalized to a value between 0 and 1).

3) Set $i=1$.

4) Input training data $\mathbf{x}_{\mathbf{i}}$ into the color recognition network. Set the teaching signal of the output layer neuron relating to the color name to the ratio of the responses of the 60 participants with normal color perception. Set the input value of the teaching signal for the neuron relating to luminance to the Y-value.

5) Activate the color recognition network. 
6) Adjust the number of connections until the error sum of squares of the teaching signal and the output value from the output layer is minimized. Use backpropagation (method of moments) in the training.

7) Set $i=i+1$, select another training data $\mathbf{x}_{\mathbf{i}}$ and then return to 4). When all of the training data has been taught, initiate the completion judgment in 8).

8) Repeat steps 3) through 7) until the total error sum of squares becomes constant. This kind of color recognition network can predict the color name and luminance of a particular color.

\section{Training the Visibility Prediction Neural Network}

Fig. 5 presents the structure of the visibility prediction network. Similar with the existing literature [7], the network is made up of three layers. The input is comprised of the features of the background colors and left and right character colors as extracted from the color recognition network, that is, the output value differences from the output layers of the three color recognition networks (the number of features being $14 \times 2$ ). In this case, the luminance value difference and the color difference of the background and character colors may influence the color scheme visibility. Therefore, this study utilizes the difference in the output values of the background color and left and right character colors from the output layer of the color recognition network as features.

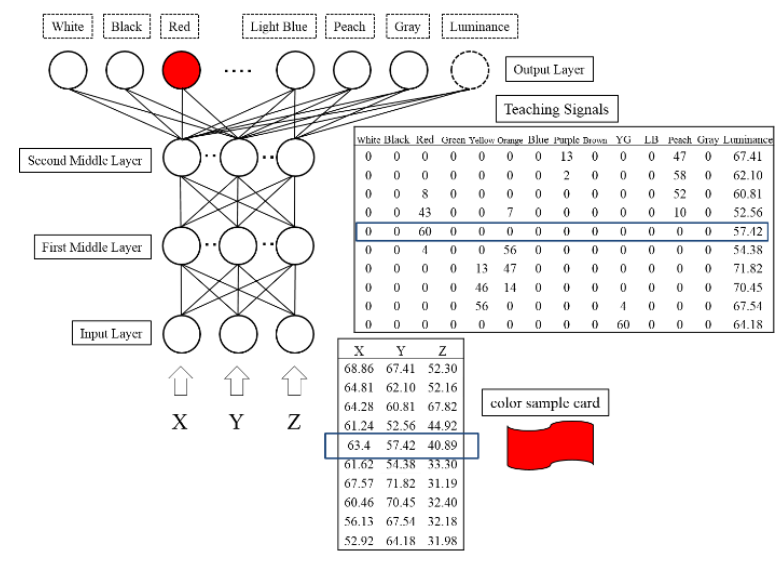

Fig. 4. Color Recognition Neural Network.

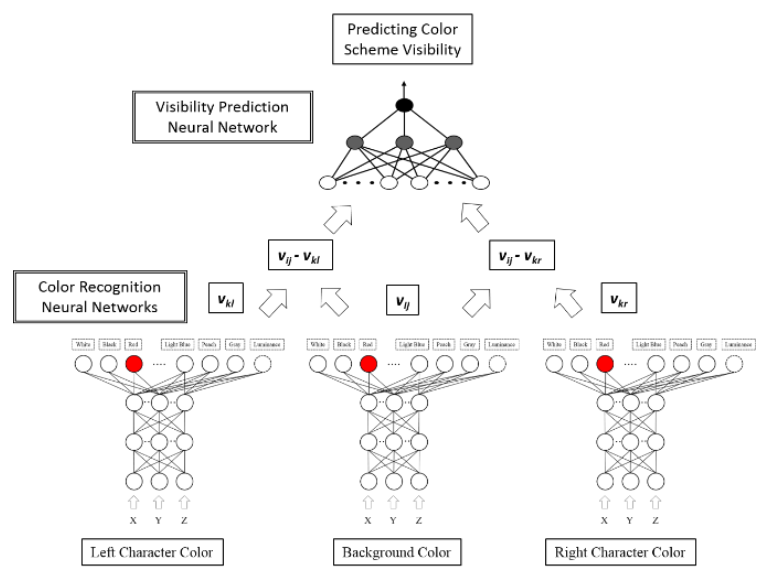

Fig. 5. Visibility Prediction Neural Network.
The number of input layer neurons for the visibility prediction network was the number of the above features. The number of middle layer neurons was set at 128 , and the number of output layer neurons was set at one. When the right character color was easier to view in combination with the background and left and right character colors, a teaching signal was applied to fire the output layer neuron (output value of 1). Moreover, when the left character was easier to view, a teaching signal was applied to avoid firing the output layer neuron (output value of 0 ). In this way, the visibility prediction network was trained in line with the teaching signal.

Additionally, this study set the observations concerning the red, blue, and green character color groupings (1200 observations) as basic color groupings taken from 4000 observations per participant gathered from the pairwise comparison experiment data outlined in Section 2.1 and used them as training data to develop the visibility prediction network. The remaining seven character color groupings (cyan, magenta, yellow, and the four WSC) were used as evaluation data (2800 observations), and the color scheme visibility prediction was performed. The learning algorithm is as follows.

1) Randomize the number of connections within the visibility prediction network.

2) Input background color $\mathrm{j}(\mathrm{j}=1,2,3,4)$ of the color group i $(i=1,2, \cdots, 10)$, and left character color $1(l=1,2,3,4,5)$ and right character color $\mathrm{r}(\mathrm{r}=1,2,3,4,5, \mathrm{l} \neq \mathrm{r})$ of the basic color group $\mathrm{k}(\mathrm{k}=1,2,3)$ into the color recognition network, activate the network, and extract the respective features $\mathbf{v}_{\mathbf{i j}}, \mathbf{v}_{\mathbf{k l}}, \mathbf{v}_{\mathbf{k r}}$ (the output values from the output layer). Set $\mathbf{v}_{\mathbf{i j k k r}}$ as the feature, which is the combination of these features. The feature to be inputted into the visibility prediction network will thus be $\mathbf{v}_{\mathbf{i j k k l}}$ $=\left(\mathbf{v}_{\mathbf{i j j}}-\mathbf{v}_{\mathbf{k l}}, \mathbf{v}_{\mathbf{i j}}-\mathbf{v}_{\mathbf{k r}}\right)^{\mathrm{T}}$.

3) Input the feature $\mathbf{v}_{\mathbf{i j k k}}$ into all of the neurons in the input layer of the visibility prediction network.

4) Set the teaching signal $t_{i j k l r}$.

5) Activate the visibility prediction network and derive the output value $\mathrm{o}_{\mathrm{ijkl} r}$.

6) Adjust the number of connections until the error sum of squares of the teaching signal $t_{i j k l r}$ and the output value $o_{i j k l r}$ is minimized. Use backpropagation (method of moments) in the training.

7) Make a new combination of background and left/right character colors and return to 2). When all of the combinations have been taught, perform the completion judgment in 8).

8) Repeat steps 2) through 7) until the total error sum of squares becomes constant.

\section{E. Predicting the Pairwise Comparison Table of an Unfamiliar Color Scheme}

Next, the trained network was used to predict the visibility of character colors that lie outside of the basic color groupings. For unfamiliar background color $j$ in color grouping $i$, and left character color $l$ and right character color $r$ both in color grouping $k$, the feature $\boldsymbol{v}_{i j k l r}$ will be extracted from the color recognition network. Thus, when the feature $v_{i j k l}$ is entered into the visibility prediction network and the network activated, the 
output value $o_{i j k l r}$ can be treated as the probability that the righthand character color will have higher visibility for the color scheme. If the output value from the visibility prediction network is the threshold (set at 0.5 or higher), then for that color scheme, the right-hand character color will be judged as easier to view. If it is less than the threshold, then the left-hand character color will be judged as easier to view.

The element for predicting the pairwise comparison tables utilized the visibility scores for the background and character colors derived by the Thurstone pairwise comparison method (Section 2.2), which employed the output values from the visibility prediction network. For example, when predicting the pairwise comparison tables for the case where the character colors are from the cyan grouping and the background color is from the red, the features of the 40 combinations of character color (5 colors, \#003333, \#006666, \#009999, \#00cccc, \#00ffff) and background color (4 colors, \#660000, \#990000, \#cc0000, \#ffff00) shown in Table I and Table II are entered into the network and 40 output values are derived. Next, taking the output value from the network, $o_{i j k l r}$, as the probability that the right-hand character was predicted as being easier to see $\left(y_{i j k l r}\right)$, the value of the inverse function of the cumulative distribution function of the standard normal distribution was derived, mean values for all colors were calculated, and the pairwise comparison scores $z_{i j k r}$ were derived. For every other color scheme, the above process was carried out in the same way for all 40 combinations to create the pairwise comparison tables. The above process yielded a total of 70 pairwise comparison tables for seven character color groupings.

\section{Evaluation}

\section{A. Evaluation Method}

The red, green, and blue character colors were set as basic color groupings, and the proposed method was constructed from the corresponding data from the pairwise comparison experiment (1200 observations). The data from the remaining seven color groupings (2800 observations) were treated as unfamiliar color schemes, and a pairwise comparison table prediction was performed.

The evaluation method was as follows. To investigate whether it was possible to use the proposed method to predict the visibility of unfamiliar color schemes, visibility predictions for these schemes were performed for each participant, the predictions were compared to the actual pairwise comparison data, and the predictive precision was derived. Similarly, to investigate whether it was possible to predict pairwise comparison tables, the pairwise comparison tables predicted by the proposed method and the tables created with actual experimental data were compared. In the final step, the validity of the proposed method was investigated by comparing the input features of the visibility prediction network as produced by the proposed method and the two methods (outline below) in the existing literature [7], [8].

- Existing Method-1 [7]: All RGB, HSV, XYZ, and $\mathrm{L}^{*} \mathrm{a} \mathrm{b}^{*}$ color system values for the background and left/right character colors were used. In this case, there were 36 features denoting that the number of neurons in the input layer of the visibility prediction network was also 36 .

- Existing Method-2 [8]: For the background and left/right character colors, in line with the Multi-Stage Color Model, response values of the LMS cones (Equation (3)), the response values $\left(\mathrm{R}_{0}, \mathrm{~B}_{0}, \mathrm{G}_{0}, \mathrm{Y}_{0}\right)$ corresponding to the red, blue, green, and yellow responses (Equation (5)), and luminance values $\mathrm{V}$ (Equation (6)) were derived and used as features. In addition, the difference in luminance between the background color and left/right character colors and the difference in the response value of the opponent colors $\left(R_{0} G_{0}, B_{0} Y_{0}\right)$ were also considered as features. In this case, there were 30 features denoting that the number of neurons in the input layer of the visibility prediction network was also 30 .

Setting the structure (number of middle and output layers) and training conditions of the visibility prediction networks, the same characters were inputted, and the visibility prediction networks were constructed (networks were constructed for each participant).

\section{B. Results of the Color Scheme Visibility Prediction}

Using the trained network, predictions on whether the left or right character would be more visible against the background were made by each participant for the seven nonbasic character color groupings. The results were then compared to the actual pairwise comparison experiment data (2800 observations). Table IV presents the results of the two existing methods and the proposed method. Furthermore, the table also shows the mean values of the predictive precision (the ratio of correct answers for the 2800 observations) for each color for the 20 participants and the mean values of the predictive precision with respect to all character colors for each of the existing and proposed methods.

Table IV shows that the mean value of the predictive precision for the 20 participants with respect to all character colors using the proposed method was $82.16 \%$. For the Existing Method-1, the result was $79.58 \%$, and for the Existing Method-2, the value was $81.52 \%$. Using the proposed method returns a level of predictive precision that is higher than the Existing Method-2 (MSC Model), which has higher predictive accuracy of the two existing methods.

TABLE. IV. RESUlTS OF THE COLOR SCHEME VISIBILITY PREDICTION

\begin{tabular}{|l|l|l|l|}
\hline Character color & $\begin{array}{l}\text { Existing } \\
\text { Method-1 }\end{array}$ & $\begin{array}{l}\text { Existing } \\
\text { Method-2 }\end{array}$ & $\begin{array}{l}\text { Proposed } \\
\text { Method }\end{array}$ \\
\hline Cyan & $86.20 \%$ & $89.97 \%$ & $88.36 \%$ \\
\hline Magenta & $82.48 \%$ & $87.55 \%$ & $86.67 \%$ \\
\hline Yellow & $88.16 \%$ & $89.71 \%$ & $89.46 \%$ \\
\hline WSC1 & $78.17 \%$ & $80.97 \%$ & $81.87 \%$ \\
\hline WSC2 & $80.65 \%$ & $78.93 \%$ & $82.02 \%$ \\
\hline WSC3 & $58.68 \%$ & $57.41 \%$ & $60.35 \%$ \\
\hline WSC4 & $82.75 \%$ & $86.08 \%$ & $86.41 \%$ \\
\hline \hline Mean & $79.58 \%$ & $81.52 \%$ & $82.16 \%$ \\
\hline
\end{tabular}




\section{Results of the Prediction of Pairwise Comparison Tables}

In this stage, pairwise comparison tables for the seven nonbasic color groupings were predicted and compared to those created from actual experimental data. As an example, Table V presents the pairwise comparison tables predicted using the proposed method for the case where the character colors were from the cyan grouping, and the background color was from the red grouping. The upper values show the actual pairwise comparison scores and the values shown in parentheses are the predicted pairwise comparison scores.

With regard to the predictive precision of the pairwise comparison tables, it is sufficient to evaluate them by using the difference between the predicted value and the actual pairwise comparison score. The evaluation index employs the error sum of squares between these two values. Specifically, as shown in Table V, when using the pairwise comparison tables to change a color scheme for easier viewing, it is sufficient to change to a color scheme with a high score. Therefore, when using pairwise comparison tables to compare color scheme visibility, the magnitude relationship of adjacent scores should be assessed. In this context, the actual and predicted values of the magnitude relationship between the score of an arbitrary color scheme in the table and the scores of its eight adjacent (above/below, left/right, and diagonal) color schemes were tested for consistency for all color schemes. The ratio of these values (referred to as the accuracy rate) was used as the predictive precision of the pairwise comparison tables. Table VI and Table VII present the accuracy rate for each of the character colors for the 20 participants as well as the mean of the error sum of squares and the mean value for all character colors for each of the existing and proposed methods.

TABLE. V. PREDiction of ThURSTONE PAIRWISE COMPARISON Method (CHARACTER COLOR CYAN, BACKGROUND COLOR RED)

\begin{tabular}{|l|l|l|l|l|l|}
\hline \multirow{2}{*}{$\begin{array}{l}\text { Background } \\
\text { color }\end{array}$} & \multicolumn{5}{|l|}{ Character colors } \\
\cline { 2 - 6 } & $\# 003333$ & $\# 006666$ & $\# 009999$ & $\# 00 \mathrm{cccc}$ & $\# 00 \mathrm{ffff}$ \\
\hline \multirow{2}{*}{$\# 660000$} & -1.352 & -0.676 & 0.071 & 0.761 & 1.196 \\
& $(-1.325)$ & $(-0.557)$ & $(-0.066)$ & $(0.718)$ & $(1.230)$ \\
\hline \multirow{2}{*}{$\# 990000$} & -0.621 & -1.163 & -0.172 & 0.723 & 1.233 \\
& $(-0.659)$ & $(-0.719)$ & $(-0.393)$ & $(0.50)$ & $(1.272)$ \\
\hline \multirow{2}{*}{$\# \mathrm{cc} 0000$} & -0.092 & -0.894 & -0.608 & 0.361 & 1.233 \\
& $(-0.179)$ & $(-0.842)$ & $(-0.613)$ & $(0.460)$ & $(1.174)$ \\
\hline \multirow{2}{*}{$\# f f 0000$} & 0.534 & -0.461 & -0.671 & -0.012 & 0.611 \\
& $(0.482)$ & $(-0.313)$ & $(-0.924)$ & $(-0.05)$ & $(0.806)$ \\
\hline
\end{tabular}

TABLE. VI. RESULTS OF THE PREDICTION OF PAIRWISE COMPARISON TABLES (ACCURACY RATE)

\begin{tabular}{|l|l|l|l|}
\hline Character color & $\begin{array}{l}\text { Existing } \\
\text { Method-1 }\end{array}$ & $\begin{array}{l}\text { Existing } \\
\text { Method-2 }\end{array}$ & $\begin{array}{l}\text { Proposed } \\
\text { Method }\end{array}$ \\
\hline Cyan & $82.28 \%$ & $86.61 \%$ & $84.55 \%$ \\
\hline Magenta & $78.39 \%$ & $82.68 \%$ & $81.50 \%$ \\
\hline Yellow & $85.21 \%$ & $87.03 \%$ & $85.84 \%$ \\
\hline WSC1 & $74.86 \%$ & $77.19 \%$ & $78.02 \%$ \\
\hline WSC2 & $75.61 \%$ & $74.14 \%$ & $76.86 \%$ \\
\hline WSC3 & $59.49 \%$ & $59.39 \%$ & $61.55 \%$ \\
\hline WSC4 & $77.70 \%$ & $81.08 \%$ & $79.81 \%$ \\
\hline \hline Mean & $76.22 \%$ & $78.30 \%$ & $78.30 \%$ \\
\hline
\end{tabular}

TABLE. VII. RESULTS OF THE PREDICTION OF PAIRWISE COMPARISON TABLES (MEAN OF THE ERROR SUM OF SQUARES)

\begin{tabular}{|l|l|l|l|}
\hline Character color & $\begin{array}{l}\text { Existing } \\
\text { Method-1 }\end{array}$ & $\begin{array}{l}\text { Existing } \\
\text { Method-2 }\end{array}$ & $\begin{array}{l}\text { Proposed } \\
\text { Method }\end{array}$ \\
\hline Cyan & 0.424 & 0.269 & 0.340 \\
\hline Magenta & 0.633 & 0.354 & 0.395 \\
\hline Yellow & 0.361 & 0.286 & 0.306 \\
\hline WSC1 & 0.748 & 0.559 & 0.543 \\
\hline WSC2 & 0.626 & 0.656 & 0.557 \\
\hline WSC3 & 1.496 & 1.568 & 1.401 \\
\hline WSC4 & 0.617 & 0.422 & 0.426 \\
\hline \hline Mean & 0.701 & 0.588 & 0.567 \\
\hline
\end{tabular}

The results in Table VI and Table VII are as follows: proposed method 78.30\% (0.567), Existing Method-1 76.22\% (0.701), and Existing Method-2 78.30\% (0.588). The accuracy rate for the proposed method was approximately the same as that for the Existing Method-2 (MSC Model), but the value of the error sum of squares shows that there has been an improvement in predictive precision. The above results indicate that, with regard to the prediction of pairwise comparison tables, the proposed method realizes an improvement in predictive precision when compared to the methods in the existing literature.

\section{DISCUSSION}

\section{A. Predictive Precision of the Color Recognition Network}

This section considers the predictive precision of the color recognition network. When actually predicting color scheme visibility, all results of the color sample cards (652 colors) were utilized in constructing the color recognition network. From the perspective of network evaluation, the color sample cards were divided into training data and evaluation data. Two patterns for the data (2-cross validation) were created, one where the even-numbered colors (of the 652 total colors) were used for training data and the odd-numbered colors were used for the evaluation data and another where the odd-numbered colors were used for the training and even numbered colors for evaluation. Under the conditions described in Section 4.3, the training data was used to develop the color recognition network. Color name prediction was performed on the evaluation data of sixty participants, and the level of predictive precision was derived. Table VIII presents the results of this process.

Table VIII presents the mean value, standard deviation, and maximum and minimum values for the accuracy rate for the sixty participants. For comparison purposes, the results of the nearest neighbor algorithm are also shown. Table VIII shows that the color recognition network can be used to predict the color recognition of individual participants with a predictive precision level of approximately $75 \%$ (compared to the nearest neighbor algorithm, an increase of $10.5 \%$ in the predictive precision level can be expected).

\section{B. Features Utilized in the Training of the Visibility Prediction Network}

In this study, the output values from the output layer of the color recognition network (referred to as Feature-1) were 
utilized as features for training the visibility prediction network. The output values of the middle layers of a network trained through backpropagation can be used as valid features after the output of output values in line with the teaching signal. In this context, the output values of every middle layer in the color recognition network were trialed as a feature in the visibility prediction network (referred to as Feature-2). In this case, given that differences in the output values of all of the middle layers of the three color recognition networks were taken as features, the number of features to be entered into the visibility prediction network was $80(40 \times 2)$.

TABLE. VIII. Predictive Precision of the Color ReCognition NETWORK

\begin{tabular}{|l|l|l|l|l|}
\hline & Mean & $\begin{array}{l}\text { Standard } \\
\text { Deviation }\end{array}$ & Maximum & Minimum \\
\hline $\begin{array}{l}\text { Proposed } \\
\text { Method }\end{array}$ & $75.47 \%$ & $3.66 \%$ & $81.90 \%$ & $65.34 \%$ \\
\hline $\begin{array}{l}\text { Nearest } \\
\text { Neighbor }\end{array}$ & $64.98 \%$ & $3.16 \%$ & $70.55 \%$ & $57.21 \%$ \\
\hline
\end{tabular}

TABLE. IX. RESULTS OF THE COLOR SCHEME VISIBILITY PREDICTION WITH FEATURE-1 AND FEATURE-2

\begin{tabular}{|l|l|l|}
\hline \multirow{2}{*}{ Character color } & Proposed Method \\
\cline { 2 - 3 } & Feature-1 & Feature-2 \\
\hline Cyan & $88.36 \%$ & $89.48 \%$ \\
\hline Magenta & $86.67 \%$ & $88.95 \%$ \\
\hline Yellow & $89.46 \%$ & $92.01 \%$ \\
\hline WSC1 & $81.87 \%$ & $83.16 \%$ \\
\hline WSC2 & $82.02 \%$ & $83.90 \%$ \\
\hline WSC3 & $60.35 \%$ & $60.96 \%$ \\
\hline WSC4 & $86.41 \%$ & $89.17 \%$ \\
\hline \hline Mean & $82.16 \%$ & $83.95 \%$ \\
\hline
\end{tabular}

TABLE. X. RESULTS OF THE PREDICTION OF PAIRWISE COMPARISON TABLES WITH FEATURE-1 AND FEATURE-2 (ACCURACY RATE AND MEAN OF THE ERROR SUM OF SQUARES)

\begin{tabular}{|l|l|l|}
\hline \multirow{2}{*}{ Character color } & \multicolumn{2}{|l|}{ Proposed Method } \\
\cline { 2 - 3 } & Feature-1 & Feature-2 \\
\hline \multirow{2}{*}{ Cyan } & $84.55 \%$ & $86.14 \%$ \\
& $(0.340)$ & $(0.30)$ \\
\hline \multirow{2}{*}{ Magenta } & $81.50 \%$ & $84.22 \%$ \\
& $(0.395)$ & $(0.317)$ \\
\hline \multirow{2}{*}{ Yellow } & $85.84 \%$ & $89.47 \%$ \\
& $(0.306)$ & $(0.217)$ \\
\hline \multirow{2}{*}{ WSC1 } & $78.02 \%$ & $79.19 \%$ \\
& $(0.543)$ & $(0.508)$ \\
\hline \multirow{2}{*}{ WSC2 } & $76.86 \%$ & $79.34 \%$ \\
& $(0.557)$ & $(0.464)$ \\
\hline \multirow{2}{*}{ WSC3 } & $61.55 \%$ & $61.80 \%$ \\
& $(1.401)$ & $(1.362)$ \\
\hline \multirow{2}{*}{ WSC4 } & $79.81 \%$ & $84.40 \%$ \\
& $(0.426)$ & $(0.325)$ \\
\hline \hline \multirow{2}{*}{ Mean } & $78.30 \%$ & $80.65 \%$ \\
& $(0.567)$ & $(0.499)$ \\
\hline
\end{tabular}

The results of the color scheme visibility and pairwise comparison table predictions after training the visibility prediction network with Feature-2 are presented in Table IX and Table $X$. The training conditions were similar for Feature-1.

Table IX shows that Feature-2 has an accuracy level of $83.95 \%$ when predicting color scheme visibility results, which is 1.8 percentage point improvement over the result for Feature-1 (82.16\%). Furthermore, Table $\mathrm{X}$ shows that using Feature 2 when predicting pairwise comparison tables yields an accuracy level of $80.65 \%$ with an error sum of squares of 0.499 , which is an improvement over the result for Features-1 (accuracy rate of $78.30 \%$, error sum of squares of 0.567 ). Accordingly, when predicting color scheme visibility, the output values of the color recognition network and the output values of the middle layer are important. Specifically, the results confirm the validity of the color recognition network.

\section{CONCLUSION AND FUTURE WORK}

Based on the research into the prediction of pairwise comparison tables as an index of the visibility of background and character colors, this study constitutes an attempt to improve the predictive precision of these tables by training a neural network capable of predicting color scheme visibility. The proposed method consists of a color recognition neural network for extracting features and a visibility prediction neural network for predicting color scheme visibility. For the feature extraction element, the data collected from the color recognition experiment was used to develop a color recognition neural network, which extracted features related to color scheme visibility. The prediction element used the extracted features to train the visibility prediction neural network. In the final step, data collected from the pairwise comparison experiment was used to predict the pairwise comparison tables of unfamiliar color schemes.

The results of this study show that the proposed method can produce higher levels of predictive precision than those used in previous studies, and that it is possible to develop a neural network that can be trained to be capable of predicting color scheme visibility. Future research plans include the collection of more data through color recognition experiments and improving the color recognition network by attempting to increase the predictive precision through the extraction of color scheme visibility features with higher validity.

\section{REFERENCES}

[1] J. Ling and P. Schaik, "The effect of text and background colour on visual search of web pages," Displays, Vol. 23, No. 5, pp.223-230, 2002.

[2] R. H. Hall and P. Hanna, "The impact of web page text-background colour combinations on readability, retention, aesthetics and behavioural intention," Behaviour and Information Technology, Vol. 23, No. 3, pp.183-195, 2004.

[3] N. Nishiuchi, K. Yamanaka, and K. Beppu, "A Study of Visibility Evaluation for the Combination of Character Color and Background Color on a Web Page," 2008 Second International Conference on Secure System Integration and Reliability Improvement, pp.191-192, 2008.

[4] D. Bhattacharyya, B. Chowdhury, T. Chatterjee, M. Pal, and D. Majumdar, "Selection of character/background colour combinations for onscreen searching tasks: An eye movement, subjective and performance approach," Displays, Vol. 35, No. 3, pp.101-109, 2014.

[5] M. Grozdanovic, D. Marjanovic, G. L. Janackovic, and M. Djordjevic, "The impact of character/backgroundcolour combinations and 
exposition oncharacter legibility and readability onvideo display units," Transactions of the Institute of Measurement and Control, Vol. 39, No. 10, pp.1454-1465, 2017.

[6] D. Saito, K. Saito, K. Notomi, and Masao Saito, "A Study on Visibility Rating of Several Representative Web-Safe Color," IEEJ Transactions on Electronics, Information and Systems, Vol. 125, No. 6, pp.892-897, 2005 (in Japanese).

[7] T. Oikawa and Y. Shinozawa, "Prediction of visibility for background and character colors on the web browser," Transactions of Human Interface Society, Vol. 14, No. 2, pp.75-86, 2012 (in Japanese).

[8] M. Yamaguchi and Y. Shinozawa, "Prediction of Visibility for Color Schemewith a Multi-stage Color Model," Transactions of Information
Processing Society of Japan, Vol. 54, No. 3, pp.1230-1241, 2013 (in Japanese).

[9] R. L. De Valois and K. K. De Valois, "A multi-stage color model," Vision Research, Vol.33, No.8, pp.1053-1065, 1993.

[10] F. Vi'enot, H. Brettel, and J. D. Mollon, "Digital video colourmaps for checking the legibility of displays by dichromats," COLOR research and application, Vol.24, No.4, pp.243-252, 1999.

[11] DIC Color Guide 19th Edition, http://www.dic-graphics.co.jp/ products/cguide/dic_color_guide.html (2019/06/17 author checked).

[12] B. Berlin and P. Kay, "Basic Color Terms: Their Universality and Evolution," Berkeley \& Los Angeles: University of California Press, 1969. 